\title{
The value of dual-source multidetector-row computed tomography in determining pulmonary blood supply in patients with pulmonary atresia with ventricular septal defect
}

\author{
N. Chaosuwannakit, P. Makarawate \\ Radiology Department, Faculty of Medicine, Khon Kaen University, Khon Kaen, Thailand \\ [Received: 12 May 2017; Accepted: 16 June 2017]
}

Background: Primary evaluation of patients with pulmonary atresia with ventricular septal defect (PA-VSD) traditionally relies upon echocardiography and conventional cardiac angiography (CCA). Cardiac angiography is considered the gold standard for delineation of anatomy in children with PA-VSD. Data comparing CCA and dual-source multidetector-row computed tomography angiography (MDCT) in PA-VSD patients is limited. The objective of this study was to test the hypothesis that MDCT is equivalent to CCA for anatomic delineation in these patients.

Materials and methods: Twenty-eight patients with PA-VSD underwent CCA and $M D C T$ in close proximity to each other without interval therapy. A retrospective review of these 28 patients was performed. All MDCT data of pulmonary artery morphology, major aortopulmonary collateral arteries (MAPCAs) and type of blood supply (dual vs. single supply) were evaluated by blinded experts and results were compared with CCA.

Results: Twenty-eight patients had adequate size right and left pulmonary arteries (21 confluent and 7 non-confluent). Seven patients had complete absence of native pulmonary artery and 3 patients had stenosis of distal branches of pulmonary arteries; all had MAPCAs from descending thoracic aorta and/or subclavian arteries. Sensitivity, specificity, positive and negative predictive value of MDCT for detecting confluent of pulmonary arteries, absence of native pulmonary artery and stenosis of pulmonary arteries were all $100 \%$. Moreover, accuracy of detecting MAPCAs was excellent.

Conclusions: These results suggest that MDCT and CCA are equivalent in their ability to delineate pulmonary artery anatomy and MAPCAs. Dual source MDCT provides high diagnostic accuracy in evaluation of pulmonary blood supply in patients with PA-VSD and allows precise characterisation of the condition of pulmonary arteries and MAPCAs which is of paramount importance in managing patients with PA-VSD. (Folia Morphol 2018; 77, 1: 116-122)

Key words: dual source multidetector row computed tomography, pulmonary atresia with ventricular septal defect, cardiac imaging 


\section{INTRODUCTION}

Pulmonary atresia with ventricular septal defect (PAVSD) belong to a group of congenital cardiac malformations characterised by the lack of luminal continuity and blood flow from either the ventricle and the pulmonary artery, together with ventricular septal defect $[5,6,10$, $17,22]$. The pulmonary circulation in PA-VSD can be supplied with native pulmonary arteries, systemic circulation from patent ductus arteriosus (PDA) or major aortopulmonary collateral arteries (MAPCAs), or a combination of the two $[6,15,22]$. Major aortopulmonary arteries are large systemic collateral arteries, typically one to six in number but sometimes more, usually originating from the descending thoracic aorta [11]. Occasionally they arise from subclavian artery, aortic arch or abdominal aorta or uncommonly from other systemic arteries such as carotid, or coronary arteries $[11,22]$. They most commonly terminate by joining an interlobar or intralobar pulmonary artery that arborizes normally within a pulmonary lobe or segment, but they distribute distally into the lungs and do not communicate with the central pulmonary artery system in some cases [11]. Most patients with PA-VSD have a diffusely enlarged network of bronchial arteries providing a nondiscrete source of the pulmonary circulation [11]. They are usually smaller and more numerous than MAPCAs [11, 22]. Nonbronchial systemic arteries, such as the paramediastinal collateral arteries and the intercostals collateral arteries, also supply the lungs [11]. The morphology of these vessels can be highly complex by nature, and they can undergo changes during the course of disease.

Some progress to stenosis and complete occlusion, and others without stenoses usually become hypertensive [3]. On the basis of the characterisation of the pulmonary circulation, PA-VSD is classified into three types [22]. In type A, the native pulmonary arteries are present and are supplied by the PDA. In type $B$, pulmonary blood flow is provided by both native pulmonary arteries and by MAPCAs. In type $C$, native pulmonary arteries are absent and the blood supply is only through MAPCAs. When surgical management of PA-VSD is possible, MAPCAs are unified at the hilum, right ventricular pulmonary arterial continuity is established, and the VSD is closed [11, 19].

Primary evaluation of patients with PA-VSD traditionally relies upon echocardiography and conventional cardiac angiography, both of which have potential limitations $[1,14]$. Echocardiography is an operator dependent and limited by small window.
Furthermore, echocardiography does not provide the surgeon with a precise anatomical roadmap. Although conventional cardiac angiography (CCA) has long been accepted for preoperative evaluation to obtain an accurate map of pulmonary circulation, it is an invasive procedure with a risk of catheter complications. Both surgery and endovascular interventions are not always feasible and are associated with complications $[3,11,19]$.

The use of multidetector-row computed tomography angiography (MDCT) for evaluation of MAPCAs has been reported with older-generation scanners using three-dimensional (3D) imaging software, a complex pulmonary blood supply can noninvasively and accurately imaged with high-resolution MDCT $[2,12,13,16]$. Precise characterisation of the condition of pulmonary arteries and MAPCAs is of paramount importance in managing patients with PA-VSD. Development of newer scanner such as dual-source MDCT uses two X-ray tubes and detectors to achieve an improved temporal resolution of $83 \mathrm{~ms}$. with a gantry rotation time of $330 \mathrm{~ms}$ with use of a single-segment reconstruction mode [20]. This newer scanner MDCT provides an alternative to alleviate these pitfalls of traditional cardiac diagnostic studies. Therefore, the purpose of the current study was to evaluate the diagnostic accuracy of dual source MDCT in the identification and the comprehensive evaluation of patients with PA-VSD compared with CCA as the standard of reference.

\section{MATERIALS AND METHODS}

\section{Patient population}

This retrospective study included 28 patients with diagnosis of PA-VSD who underwent both MDCT and CCA, between February 2013 and December 2014. The indications for MDCT were preoperative evaluation of PA-VSD patients which is considered appropriate indications for MDCT, based on the criteria of the American College of Cardiology (ACC) [21]. Exclusion criteria for MDCT included the presence of renal failure, and a history of allergic reaction to iodine-containing contrast agents.

\section{Dual-source MDCT scanning protocol}

Short-term IV sedation is often required, particularly in children under 5 years of age, and the imaging can be performed during quiet breathing. Imaging was performed by using a dual-source MDCT scanner (Somatom Definition; Siemens Healthcare, Forchheim, 


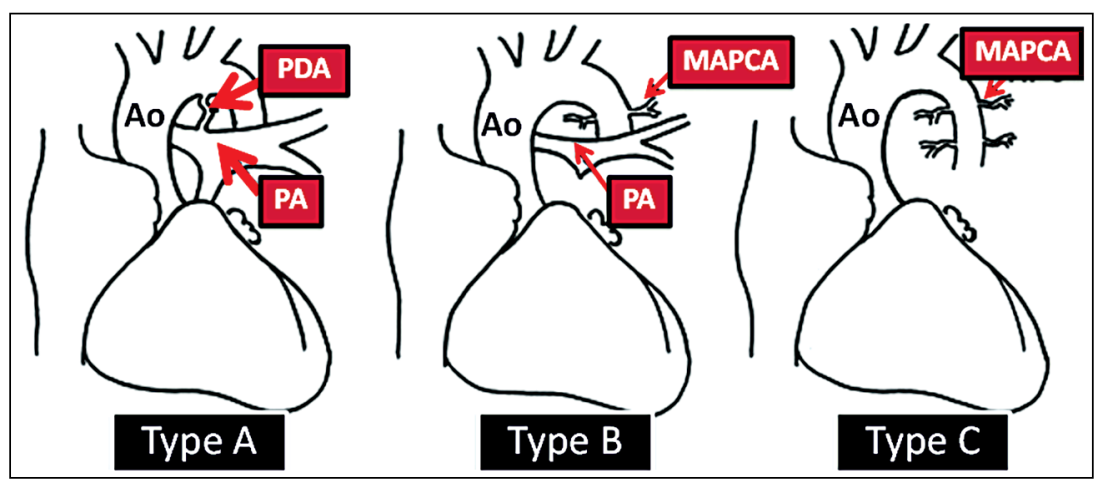

Figure 1. Illustration demonstrates classification of pulmonary atresia (PA) with ventricular septal defect according to the native $P A$, patent ductus arteriosus (PDA), and major aortopulmonary collateral artery (MAPCA); Ao — aorta; Type $\mathbf{A}$ - Native pulmonary artery present feeding by PDA; Type B — Native pulmonary artery and MAPCA present; Type C — Absence of native pulmonary artery, only MAPCA maintains pulmonary blood flow.

Germany). The radiation dose is kept to minimum by reducing the kilovoltage and tube current appropriately. For children weighing less than $10 \mathrm{~kg}, 10-19 \mathrm{~kg}$ and $20-30 \mathrm{~kg}$, we use $80 \mathrm{kV}$ and $80 \mathrm{mAs}, 80 \mathrm{kV}$ and $100 \mathrm{mAs}$, and $100 \mathrm{kV}$ and $120 \mathrm{mAs}$, respectively. Other cardiac computed tomography angiographic parameters were as follows: number of $\mathrm{X}$-ray tubes, two; collimation, 128 detector rows of $0.5 \mathrm{~mm}$ each, with double sampling by using rapid alteration of the focal spot in the longitudinal direction ( $z$ flying focal spot) [7]. Prior to scanning, the pitch was set automatically by the scanner software. Depending on heart rate, pitch was set between 0.2 and 0.43 . Automated dose regulation methods such as CARE dose 4D (Siemens Healthcare) may be used to reduce the radiation.

Image acquisition was performed during inspiratory breath-hold for older children or adult. To familiarise the patient with the protocol, breath-holding was practiced before the examination. A bolus of iodinated contrast material $(350 \mathrm{mg} / \mathrm{mL}$, Omnipaque; GE Healthcare) at a dose of $1.5 \mathrm{~mL} / \mathrm{kg}$ with dualhead power injector at a rate of $1.5-2.0 \mathrm{~mL} / \mathrm{s}$ for a 22-gauge cannula, $3.0 \mathrm{~mL} / \mathrm{s}$ for a 20 -gauge cannula and $4.0-5.0 \mathrm{~mL} / \mathrm{s}$ for a 18 -gauge cannula followed by a $10-20 \mathrm{~mL}$ of saline flush at a same rate to that of the contrast injection. For timing purposes, an automated bolus-tracking software was used, starting the scan automatically $6 \mathrm{~s}$ after contrast agent density in the descending aorta reached a predefined threshold of $130 \mathrm{HU}$ [4]. The entire volume of the heart and pulmonary arteries was covered during one breath-hold in approximately $5 \mathrm{~s}$ with simultaneous recording of the electrocardiography trace. Patients were scanned in the supine position. MDCT is performed from the thoracic inlet level to $L 1-L 2$. When there is suspicion of anomalous pulmonary venous drainage, the scan can be extended down to the lower border of the liver.

\section{MDCT image analysis}

MDCT image analysis was performed by two cardiovascular and thoracic radiologists in consensus (with a respective 11 and 10 years of experience in examining cardiovascular and thoracic computed tomography scans) and blinded to the clinical data and the results of CCA data. First, axial all image data are evaluated using a 3D post processing workstation with Syngo software (Siemens Healthcare). Various image reformatting techniques including curved planar reconstruction, maximum intensity projection (MIP), minimum intensity projection, and volume-rendering technique (VRT) are used to get all the clinically relevant information. Curved planar reformatting and MIP are primarily used to evaluate curved structures such as the pulmonary arteries and MAPCAs. Minimum intensity projection is used to evaluate the airway and lung parenchyma. For 3D reformatting of the complex anatomy, the VRT is used. Thin-section multiplanar reformatting is used for quantitative analysis of the structure in question.

On the basis of the characterisation of the pulmonary circulation, PA-VSD is classified into three types. In type $A$, the native pulmonary arteries are present and are supplied by the PDA. In type B, pulmonary blood flow is provided by both native pulmonary arteries and by MAPCAs. In type C, native pulmonary arteries are absent and the blood supply is only through MAPCAs [11, 17, 22] as illustration shown on Figure 1. 


\section{Conventional coronary angiography}

Conventional coronary angiography was performed according to standard techniques. Images were evaluated by an experienced cardiologist blinded to MDCT results using a validated and dedicated quantitative coronary angiography (QCA) software package (CA-CMS, QAngioXA 6.0, Medis Medical Imaging Systems, Leiden, The Netherland).

\section{Statistical analysis}

Sensitivity, specificity, and positive predictive value (PPV) and negative predictive value (NPV), including $95 \%$ confidence intervals $(\mathrm{Cl})$, for the detection of $>$ $50 \%$ luminal narrowing of pulmonary artery were considered positive ( $>50 \%$ luminal narrowing) on CCA. Continuous data were expressed as mean \pm standard deviation (SD). Statistical analyses were performed using SPSS software version 16 (SPSS, Inc., Chicago, IL, USA). A significance level of $p<0.05$ was considered a statistically significant result and all reported $p$-values were two-sided. Means were compared using unpaired t-test, and Mann-Whitney rank sum was used when data was not normally distributed.

\section{RESULTS}

Patient clinical characteristics are presented in Table 1. Twenty-eight patients ( 10 boys, 18 girls; mean age, $9 \pm 8$ years; range, 3-14 years) were included in this study over a period of 22 months. The mean time interval between MDCT and CCA was $3 \pm 10$ days (range, 2-21 days) and there were no clinical events between the two studies in any patient. In most patients (25/28), MDCT was performed before CCA, and in 3 patients, CCA was performed first.

In patient analysis, out of 28 MDCT examinations, none of them was of non-diagnostic image quality. CCA identified 20 patients with confluent pulmonary arteries. All patients (100\%) were correctly identified by MDCT. In addition, in a total of 28 patients, MDCT correctly identified the presence of non-confluent pulmonary arteries in 8 patients. Importantly, no patients with non-confluent pulmonary arteries were missed by MDCT. Therefore, the sensitivity and specificity for the detection of confluent or non-confluent pulmonary arteries were all $100 \%$. In addition, PPV and NPV were all $100 \%$.

Five patients had complete absence of native pulmonary artery and 3 patients had stenosis of proximal branches of pulmonary arteries; all had MAPCAs from descending thoracic aorta and/or subclavian arteries.
Table 1. Patient characteristics $(n=28)$

\begin{tabular}{lc}
\hline Characteristic & Value; mean \pm SD (range) \\
\hline Age [years] & $7 \pm 8(2-14)$ \\
Men & $10(35.7 \%)$ \\
Height $[\mathrm{cm}]$ & $110.8 \pm 13$ \\
Weight $[\mathrm{kg}]$ & $19 \pm 5$ \\
Body mass index $\left[\mathrm{kg} / \mathrm{m}^{2}\right]$ & $15.7 \pm 5(14-24)$ \\
\hline SD - standard deviation &
\end{tabular}

In a total of 28 patients, 3 patients were correctly classified as PA-VSD type A on MDCT (Fig. 2, true positive), whereas 20 patients were correctly classified as PA-VSD type B on MDCT and 5 patients were correctly classified as PA-VSD type C on MDCT. Consequently, the sensitivity, specificity, PPV and NPV for the classification of PA-VSD were all $100 \%$.

For pulmonary collateral vessels evaluated, no statistical significant of a total of 84 collateral vessels were identified on MDCT and 85 collateral vessels were identified on CCA $(p=0.78)$.

MDCT was equivalent to CCA to identification of PDA, 3 PDA were identified on both MDCT and CCA $(p=1)$. No statistical significant of MDCT and CCA to identified MAPCAs (MDCT $=81$ vessels, $C C T=82$ vessels, $p=0.86$ ). The MAPCA was incorrectly identified by MDCT in only 1 vessel resulting in a sensitivity and specificity of $98.7 \%$ and $100 \%$, respectively, and PPV and NPV were $100 \%$ and $98.7 \%$, respectively. The diagnostic accuracy for the detection of confluent pulmonary arteries, PDA and MAPCAs as Table 2.

\section{DISCUSSION}

The present study demonstrated excellent diagnostic accuracy for the comprehensive assessment of patient with PA-VSD using dual-source MDCT. Importantly, no patients with absence of native pulmonary artery or non-confluent pulmonary artery were missed using dual-source MDCT.

Furthermore, the excellent sensitivity, specificity, PPV and NPV suggests that MDCT might be particularly valuable in the evaluation of pulmonary collateral vessels such as PDA or MAPCAs compared with conventional cardiac angiography. These results are in line with previous published data on the performance of the older generation computed tomography scanner $[2,12,13,16]$.

Analysis of the pulmonary artery anatomy is the most important aspect of diagnostic imaging of patients 

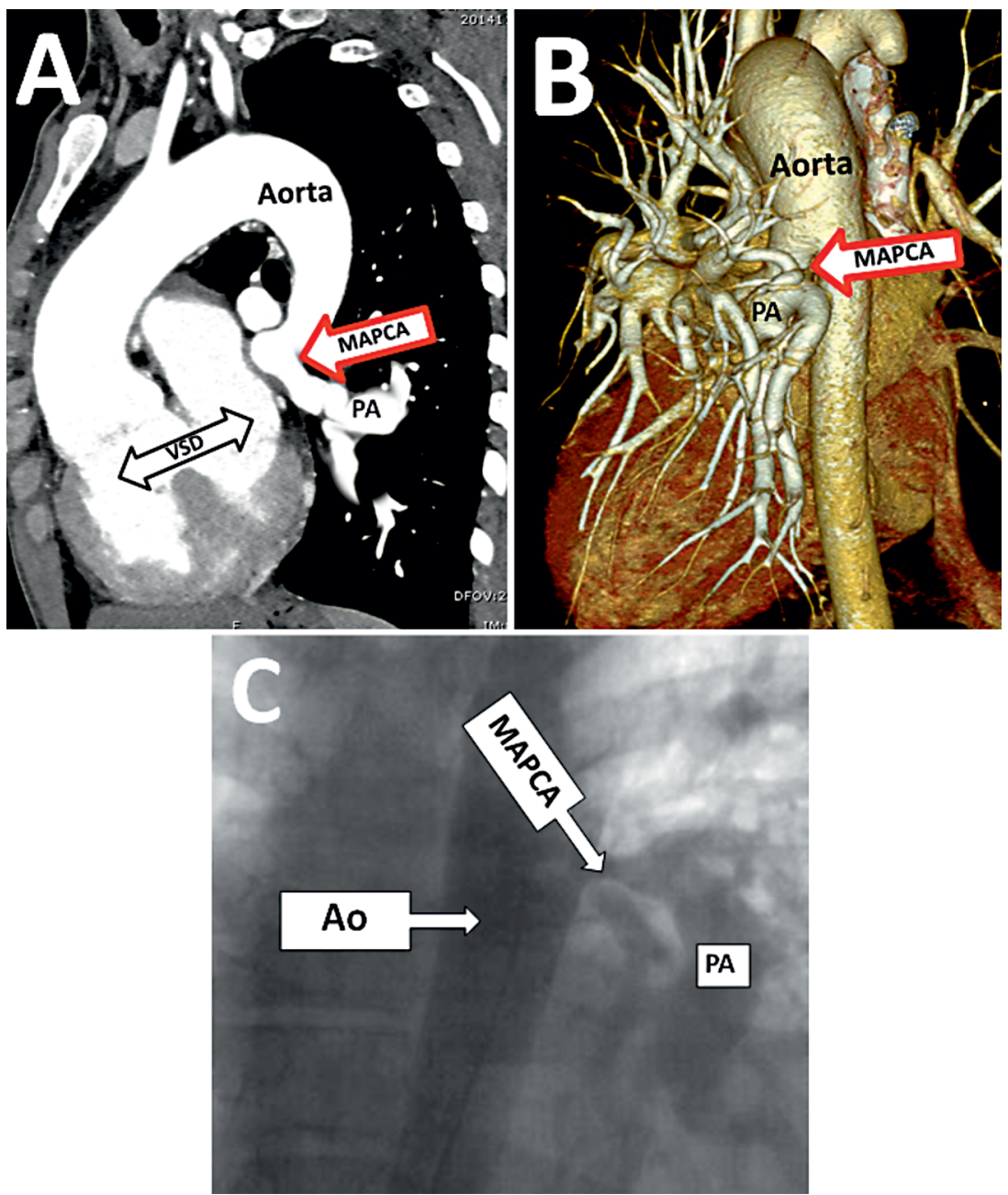

Figure 2. A 10-year-old boy with pulmonary atresia with ventricular septal defect (VSD). Dual-source multidetector computed tomography (A, B) image shows the major aortopulmonary collateral artery (MAPCA) supplying the left lung. Conventional cardiac angiography (CCA) (C) confirms the large MAPCA from descending thoracic aorta $(\mathrm{Ao})$ feeding left pulmonary artery (PA).

Table 2. Diagnostic accuracy of dual-source multidetector computed tomography for pulmonary atresia with ventricular septal defect patients compared with conventional cardiac angiography

\begin{tabular}{lccc}
\hline & Confluent PA & PDA & MAPCAs \\
\hline Sensitivity & $20 / 20(100 \%)$ & $3 / 3(100 \%)$ & $81 / 82(98.7 \%, 95-99)$ \\
Specificity & $8 / 8(100 \%)$ & $25 / 25(100 \%)$ & $81 / 81(100 \%)$ \\
Positive predictive value & $20 / 20(100 \%)$ & $3 / 3(100 \%)$ & $81 / 81(100 \%)$ \\
Negative predictive value & $8 / 8(100 \%)$ & $25 / 25(100 \%)$ & $81 / 82(98.7 \%, 95-99 \%)$ \\
\hline
\end{tabular}

Data are absolute values used to calculate percentages. Data in parentheses are percentages with 95\% confidence intervals; MAPCA — major aortopulmonary collateral artery; PA — pulmonary artery; PDA — patent ductus arteriosus

with PA-VSD. Various studies have shown that MDCT is an accurate tool for evaluating pulmonary arteries $[4,12,13]$. The presence or absence of native pulmonary artery, length of the pulmonary atresia, confluence of native main pulmonary arteries, size of main, right, and left pulmonary arteries at the origin and at pre- branching site were recorded. Images were carefully analysed for branch pulmonary artery stenosis [18].

In patients with PA-VSD, the lungs can derive their blood supply from various sources [11, 17, 22]. MAPCAs represent fetal primitive intersegmental arteries that originate from the descending aorta and have 
not involuted. They gain access to the lung through the hilum and connect with native pulmonary arteries in the mediastinum or at the lobar or subsegmental level. MAPCAs are different from bronchial arteries in a number of ways [8]. Usually, MAPCAs do not branch in their mediastinal course, whereas bronchial arteries provide mediastinal branches and, in some cases, branches to the spinal cord. MAPCAs anastomose with the intrapulmonary arteries typically at or near the pulmonary hilum instead of in the periphery. MAPCAs may accompany bronchi but do not form a nutritive plexus around bronchi. Unlike bronchial arteries, MAPCAs are virtually never connected to intercostals arteries. Most MAPCAs have an elastic wall structure resembling the pulmonary artery or aorta rather than bronchial arteries. MDCT with 3D VRT has the potential to elucidate all the MAPCAs shown by invasive catheter angiography $[8,9,16]$. Using various MDCT post processing techniques, the total number of MAPCAs and their origin, course, and intrapulmonary connections can be easily assessed. PDA, if present, is an important source of pulmonary blood supply. When ductus arteriosus is patent, generally the native pulmonary arteries will be of adequate size. When a PDA connects with a central pulmonary artery, the peripheral distribution is normal, and there are no MAPCAs in that hemithorax. Thus, a PDA does not coexist with MAPCAs in the same lung [22]. Unlike MAPCAs, the ductus does not branch before joining the central pulmonary arteries, and it tends to be less tortuous. There is a high risk of stenosis of the pulmonary arteries at the PDA insertion site.

Clinical progress by the development of MDCT technology beyond 4 slices can more likely be expected from increased temporal resolution which reduces cardiac motion artifacts and increased spatial resolution rather than from only increase in the volume coverage speed. The limitations of earlier MDCT systems in the assessment of cardiac and pulmonary arteries are the consequence of partial volume effects and beam-hardening artifacts which could be overcome by a higher isotropic spatial resolution. The total scanning time for a dual-source MDCT is shortened to approximately $5 \mathrm{~s}$. Accordingly, faster image acquisition allows for a reducing the volume of contrast media and breath-hold time causing reducing respiratory motion artifacts, and making the technique more robust $[4,7,8,9,18]$.

Despite promising initial results, our study has potential limitations. First, since our method of en- rollment included some patients who underwent CCA because of MDCT results, selection bias in a proportion of patients may have overestimated the calculated sensitivity of MDCT. However, selection bias tends to underestimate specificity, which was still high in our study, and may have had minimal effect on PPV and NPV because patient enrollment after initial MDCT was independent of the actual true disease status on CCA. Secondly, the present result is limited by the number of patients, therefore, the interpretation of sensitivity may be limited. Thirdly, our results represent a single-center experience, the generalisability of the present results is limited.

\section{CONCLUSIONS}

Dual-source MDCT provides a comprehensive evaluation of different anatomic structures, including the heart, pulmonary and systemic thoracic vasculature when evaluating patients with PA-VSD. In a clinical setting, MDCT has proved to be an invaluable diagnostic and decision-making tool as a compliment to echocardiography and increasingly as a substitute for invasive angiography. The advantage of MDCT compared with CCA is that it is noninvasive, allows avoiding most catheter-associated risks and discomforts with the exception of exposure to iodinated contrast agents and radiation. With rapidly improving technology, MDCT may well evolve from a useful complement to CCA to a clinically viable alternative.

\section{REFERENCES}

1. Acherman RJ, Smallhorn JF, Freedom RM. Echocardiographic assessment of pulmonary blood supply in patients with pulmonary atresia and ventricular septal defect. J Am Coll Cardiol. 1996; 28(5): 1308-1313, doi: 10.1016/ S0735-1097(96)00302-6, indexed in Pubmed: 8890831.

2. Bean MJ, Pannu H, Fishman EK. Three-dimensional computed tomographic imaging of complex congenital cardiovascular abnormalities. J Comput Assist Tomogr. 2005; 29(6): 721-724, indexed in Pubmed: 16272839.

3. Brown SC, Eyskens B, Mertens L, et al. Percutaneous treatment of stenosed major aortopulmonary collaterals with balloon dilatation and stenting: what can be achieved? Heart. 1998; 79(1): 24-28, indexed in Pubmed: 9505914.

4. Cademartiri F, Nieman K, van der Lugt A, et al. Intravenous contrast material administration at 16-detector row helical CT coronary angiography: test bolus versus bolus-tracking technique. Radiology. 2004; 233(3): 817-823, doi: 10.1148/radiol.2333030668, indexed in Pubmed: 15516601.

5. Diethelm E, Soto B, Nath PH, et al. The pulmonary vascularity in patients with pulmonary atresia and ventricular septal defect. RadioGraphics. 1985; 5(2): 243-254, doi: 10.1148/radiographics.5.2.243. 
6. Ellis K. The bronchial arteries and anomalous ystemic arteries to the lungs in congenital heart and lung disease. In: Butler J. The bronchial circulation. Marcel Dekker, New York, NY 1992: 599-748.

7. Flohr TG, Stierstorfer $\mathrm{K}$, Ulzheimer $\mathrm{H}$, et al. Image reconstruction and image quality evaluation for a 64-slice CT scanner with z-flying focal spot. Med Phys. 2005; 32: 2536-2547.

8. Goo HW, Park IS, Ko JK, et al. CT of congenital heart disease: normal anatomy and typical pathologic conditions. Radiographics. 2003; 23 Spec No: S147-S165, doi: 10.1148/ rg.23si035501, indexed in Pubmed: 14557509.

9. Greil GF, Kuettner A, Schoebinger M, et al. Visualization of peripheral pulmonary artery stenosis using high-resolution multidetector computed tomography. Vasc Med. 2005; 10(3): 235-236, doi: 10.1191/1358863x05vm613xx, indexed in Pubmed: 16235778.

10. Greil GF, Schoebinger M, Kuettner A, et al. Imaging of aortopulmonary collateral arteries with high-resolution multidetector CT. Pediatr Radiol. 2006; 36(6): 502-509, doi: 10.1007/ s00247-006-0143-0, indexed in Pubmed: 16555039.

11. Kouchoukos NT, Blackstone EH, Doty DB, Hanley FL, Karp RB. Ventricular septal defect with pulmonary stenosis or atresia. In: (eds) Cardiac surgery: morphology, diagnostic criteria, natural history, techniques, results and indications. 3rd edition. Elsevier, Philadelphia 2003: 946-1073.

12. Le Bret $E$, Macé $L$, Dervanian $P$, et al. Images in cardiovascular medicine. Combined angiography and three-dimensional computed tomography for assessing systemic-to-pulmonary collaterals in pulmonary atresia with ventricular septal defect. Circulation. 1998; 98(25): 2930-2931, indexed in Pubmed: 9860797.

13. Lee T, Tsai IC, Fu YC, et al. Using multidetector-row CT in neonates with complex congenital heart disease to replace diagnostic cardiac catheterization for anatomical investigation: initial experiences in technical and clinical feasibility. Pediatr Radiol. 2006; 36(12): 1273-1282, doi: 10.1007/ s00247-006-0315-y, indexed in Pubmed: 17036235.

14. Mackie AS, Gauvreau K, Perry SB, et al. Echocardiographic predictors of aortopulmonary collaterals in infants with tetralogy of fallot and pulmonary atresia. J Am Coll Cardiol. 2003; 41(5): 852-857, indexed in Pubmed: 12628734.

15. Maeda E, Akahane M, Kato N, et al. Assessment of major aortopulmonary collateral arteries with multidetectorrow computed tomography. Radiat Med. 2006; 24(5):
378-383, doi: 10.1007/s11604-006-0037-y, indexed in Pubmed: 16958418.

16. Murai S, Hamada S, Yamamoto S, et al. Evaluation of major aortopulmonary collateral arteries (MAPCAs) using three-dimensional CT angiography: two case reports. Radiat Med. 2004; 22(3): 186-189, indexed in Pubmed: 15287535.

17. O'Leary PW, Edwards WD, Julsrud PR, Puga FJ. Pulmonary atresia and ventricular septal defect. In: Allen HD, Driscoll DJ, Shaddy RE, Feltes TF. Moss and Adam's heart disease in infants, children, and adolescents. Lippincott Williams \& Wilkins, Baltimore, MD 2008: 878-88.

18. Oguz B, Haliloglu M, Karcaaltincaba M. Paediatric multidetector $\mathrm{CT}$ angiography: spectrum of congenital thoracic vascular anomalies. Br J Radiol. 2007; 80(953): 376-383, doi: 10.1259/bjr/47124005, indexed in Pubmed: 16687462.

19. Reddy VM, McElhinney DB, Amin Z, et al. Early and intermediate outcomes after repair of pulmonary atresia with ventricular septal defect and major aortopulmonary collateral arteries: experience with 85 patients. Circulation. 2000; 101(15): 1826-1832, indexed in Pubmed: 10769284.

20. Ropers U, Ropers D, Pflederer T, et al. Influence of heart rate on the diagnostic accuracy of dual-source computed tomography coronary angiography. J Am Coll Cardiol. 2007; 50(25): 2393-2398, doi: 10.1016/j. jacc.2007.09.017, indexed in Pubmed: 18154964.

21. Taylor AJ, Cerqueira M, Hodgson JM, et al. ACCF/SCCT/ACR/ AHA/ASE/ASNC 2010 Appropriate Use Criteria for Cardiac Computed Tomography: a report of the American College of Cardiology/ Foundation Appropriate Use Criteria Task Force Society of Cardiovascular Computed Tomography/ American College of Radiology/ American Heart Association/ American Society of Echocardiography/ American Society of Nuclear Cardiology/ Society for Cardiovascular Angiography and Interventions/ Society for Cardiovascular Magnetic Resonance. J Am Coll Cardiol. 2010; 56(22): 1864-1894, doi: 10.1016/j.jacc.2010.07.005, indexed in Pubmed: 21087721.

22. Tchervenkov Cl, Roy N. Congenital Heart Surgery Nomenclature and Database Project: pulmonary atresia - ventricular septal defect. Ann Thorac Surg. 2000; 69(4 Suppl): S97-105, indexed in Pubmed: 10798421. 